\title{
Éléments de réflexion pour une véritable politique de décentralisation au Québec, associée à une politique nationale des territoires
}

\author{
Bernard Vachon ${ }^{1}$, Ph. D. \\ Université du Québec à Montréal
}

\section{Mise en contexte}

Le débat sur la décentralisation ne date pas d'aujourd'hui au Québec. La question a été sérieusement mise de l'avant et envisagée comme projet majeur de réorganisation de l'État avec l'accession du Parti québécois au pouvoir en 1976. Une des premières actions d'envergure de ce gouvernement, après l'élaboration et l'adoption de la Loi sur la protection du territoire agricole (décembre 1978), a été la réforme municipale marquée principalement par la Loi sur l'aménagement et l'urbanisme (novembre 1979) et la création des MRC. Celles-ci étaient destinées à devenir les lieux privilégiés d'accueil des pouvoirs décentralisés vers les collectivités territoriales. Depuis, soit après plus de trente ans, la décentralisation ne parvient pas à déborder les frontières sécurisantes du débat intellectuel et politique. Le fossé entre la décentralisation virtuelle et la décentralisation réelle ne cesse de s'approfondir.

\section{Le fossé entre la décentralisation virtuelle et la décentralisation réelle ne cesse de s'approfondir.}

Entre-temps, plusieurs États modernes démocratiques et d'économie libérale ont résolument pris le train de la décentralisation et marquent aujourd'hui un état d'avancement qui ne souligne que davantage le retard pris par le Québec en ce domaine. Parmi les pays dont les gouvernements sont les plus décen
La décentralisation n'est pas une fin en soi, mais un mode de gouvernance qui accorde un rôle accru aux instances régionales et locales dans la prise de décisions qui concernent les enjeux économiques, sociaux, culturels et environnementaux des communautés qu'elles regroupent.

tralisés, mentionnons la Suisse, la Suède, le Danemark, l'Allemagne, l'Espagne, l'Italie, et la France. La décentralisation n'est pas le propre des pays industrialisés et riches puisqu'elle est sérieusement implantée ou en voie de l'être dans plusieurs pays d'Amérique latine (Brésil, Mexique, Chili, Bolivie, etc.) et d'Afrique (Sénégal, Mali, Burkina Faso, etc.).

Rappelons que la décentralisation n'est pas une fin en soi mais un mode de gouvernance qui accorde un rôle accru aux instances régionales et locales dans la prise de décisions qui concernent les enjeux économiques, sociaux, culturels et environnementaux des communautés qu'elles regroupent. La véritable décentralisation est étroitement associée aux efforts de modernisation de l'organisation de l'État et des structures de la société. Parce que la décentralisation accorde de réels pouvoirs aux collectivités territoriales (régions, MRC, grandes villes, municipalités rurales, dans le cas du Québec) pour déterminer et gérer leur développement, elle est l'expression d'une démocratie participative évoluée, chaque 
palier de gouvernement exerçant les compétences qui lui sont le plus appropriées en regard de la diversité des services à rendre à la population (principe de subsidiarité).

Lorsque l'on constate les progrès de la décentralisation administrative et politique réalisés depuis les années 80 dans un très grand nombre de pays et particulièrement dans des pays qui partagent notre niveau de développement et nos aspirations démocratiques, il est désolant de suivre la rhétorique grandiloquente de nos hommes et femmes politiques en ce domaine et d'observer la pauvreté, l'errance débridée, des actions prises par leur gouvernement pour en assurer la concrétisation. Les causes? Elles sont multiples. J'en relèverai sept qui m'apparaissent déterminantes :

- l'absence d'une vision claire de la décentralisation au sein des gouvernements qui se sont succédés et d'un portrait net des mérites et avantages de sa concrétisation, ce qui contribue à expliquer le peu d'enthousiasme et d'empressement à mettre en œuvre une telle politique;

- la domination d'une culture de gouvernance centralisée, bien enracinée dans l'administration publique, qui oppose une résistance farouche à l'instauration d'une véritable politique de décentralisation qui aurait pour effet de réduire le pouvoir central au profit des collectivités territoriales;

- la méfiance des élus locaux aggravée par des réformes passées usurpant le nom de décen tralisation alors qu'elles avaient pour premier but de délester l'État de certaines tâches sans contrepartie financière pour les collectivités territoriales à qui elles étaient attribuées (la réforme Ryan en est un bel exemple);

- le manque de confiance de l'État central envers la capacité de vision et de gestion des élus locaux des villes petites et moyennes, des municipalités rurales et des MRC, et de leur capacité à travailler efficacement dans un contexte de responsabilités accrues et d'un nouveau rapport avec le gouvernement central;

- la méconnaissance assez généralisée au sein de la population des « pourquoi? », « pour qui? » et « comment? » la décentralisation, ce qui explique le peu de mobilisation provenant de la base, ceci facilitant et confortant d'autant les positions centralisatrices de l'État;

- l'incapacité des grandes associations municipales d'unir leurs forces pour élaborer leur propre projet de décentralisation et amener le gouvernement à en discuter et négocier le contenu. Edgar Pisani, figure politique marquante en France au cours des années 60 et 70 écrivait: « La véritable décentralisation n'est jamais donnée mais conquise! »;

- l'étonnante habilité des gouvernements, depuis le début des années 80 , à entretenir auprès des groupes concernés et de la population en général le « rêve » de la décentralisation, ainsi qu'à inventer et à mettre en œuvre diverses formules simulacres de décentralisation dépourvues des réels pouvoirs et des ressources appropriées.

\section{Dynamique territoriale et décentralisation : un arrimage profitable}

Il est bon de se rappeler que le nouveau système de création de la richesse est à la fois localisé et planétaire, c'est-à-dire que si les marchés sont mondialisés, la production a un ancrage local. L'économie mondiale dicte les lois de la concurrence, mais c'est à l'échelon local et régional que se regroupent les conditions de la compétitivité : services aux entre- 
prises, main-d'œuvre qualifiée, structure d'organisation de la production, maîtrise et diffusion de l'innovation, etc.

Si les facteurs d'ordre économique tels le capital, les ressources naturelles, les équipements et infrastructures de transport et de communication, les marchés... continuent d'être des éléments importants dans le processus de recomposition des territoires, le paradigme renouvelé de développement accorde un rôle tout aussi important aux facteurs non économiques telle la qualification individuelle et collective, la transmission des savoirs et savoir-faire traditionnels, le cadre de vie, la perméabilité à l'innovation, la vitalité communautaire, l'ouverture à la concertation et au partenariat.

L'économie mondiale dicte les lois de la concurrence, mais c'est à l'échelon local et régional que se regroupent les conditions de la compétitivité : services aux entreprises, main-d'œuvre qualifiée, structure d'organisation de la production, maîtrise et diffusion de l'innovation, etc.

L'espace économique est ainsi enchevêtré au système social. Pour redynamiser et développer le domaine économique, il apparaît absolument nécessaire de recourir à des mesures d'ordre social, culturel et environnemental, car les actions appliquées aux seuls facteurs de production ne concernent qu'un volet de la dynamique territoriale susceptible de générer et de porter le développement. On ne peut faire fleurir des entreprises dans un désert social et culturel.

Pour redynamiser et développer le domaine économique, il apparaît absolument nécessaire de recourir à des mesures d'ordre social, culturel et environnemental.
Les analyses confirment que si l'offre de telle infrastructure ou de telle aide financière joue un rôle important dans un processus de développement, c'est davantage l'existence, au niveau des collectivités territoriales, de facteurs divers, économiques, sociaux et environnementaux et leur interaction en modes originaux d'organisation que constituent les systèmes de production, qui est déterminante. En d'autres termes, il convient de considérer la dynamique productive d'un territoire comme le résultat d'un processus de construction issu de stratégies d'acteurs économiques et sociaux et de phénomènes d'apprentissage collectif et non comme une donnée a priori ou importée. Ce qui est à la base du concept et des politiques de compétitivité territoriale.

Partant, on peut considérer que les milieux locaux et régionaux ont la possibilité, voire la responsabilité de promouvoir, de susciter, de «construire » la dynamique territoriale qui fera surgir les initiatives de développement génératrices d'emplois et de richesses. Pour y parvenir, ils auront besoin du soutien de l'État contenu notamment dans une vigoureuse politique de développement régional.

En effet, pour agir adéquatement et efficacement, les milieux locaux et régionaux devront disposer de pouvoirs et de ressources appropriés. Une volonté gouvernementale d'affirmer le rôle des collectivités territoriales en matière de développement économique et social devra conduire à l'adoption d'une politique de décentralisation des pouvoirs et des ressources.

Le Québec vit depuis plusieurs décennies dans une dialectique constante entre une décentralisation réelle et une décentralisation virtuelle. Alors que le développement territorial et la décentralisation se trouvent liés, tout projet sérieux de développement régional doit envisager accroître les pouvoirs et les moyens des acteurs politiques (imputables) des territoires. 
Le premier défi d'un gouvernement central en matière de développement économique et d'emploi est d'aider, par des actions appropriées et bien ciblées, les communautés locales et régionales à se mettre en «état de produire et de se développer $»$.
La décentralisation, qui accroît l'autonomie des territoires en matière de développement économique et social et stimule les initiatives des acteurs locaux en ces domaines, va de pair avec la dynamique territoriale et les stratégies de développement à promouvoir.

\section{Décentralisation et politique vigoureuse de développement régional}

La décentralisation n'est pas la panacée et le remède absolu aux difficultés qui affligent les régions éloignées et intermédiaires. Sans l'appui d'une véritable politique nationale de développement des territoires, les nouveaux pouvoirs des collectivités territoriales acquis par la décentralisation risquent d'avoir bien peu d'effet sur le développement économique et social.

\subsection{Mais pourquoi promouvoir le développement des régions?}

Face aux difficultés économiques et sociales que traversent les régions périphériques et intermédiaires du Québec, certains n'hésitent pas à poser le problème sous la forme des interrogations suivantes :

- Pourquoi résister au déclin économique et démographique des régions éloignées et intermédiaires?

- Pourquoi ne pas s'accommoder à l'idée de régions désertées, vidées de leurs activités et de leurs populations, abandonnées aux grands espaces sauvages offerts aux citadins comme lieux de récréation et de villégiature?

- Pourquoi ne pas laisser les règles de l'économie organiser seules la mosaïque du peuplement et la distribution des activités de production?

- Pourquoi ne pas aller dans le sens d'une concentration plus poussée de la population et des activités économiques sur les grandes zones métropolitaines du Québec : Montréal, Québec, Trois-Rivières, Hull-Gatineau?
- Pourquoi ne pas souscrire à l'idée que les régions qui survivront seront celles qui adhéreront pleinement aux conditions de la croissance industrielle et métropolitaine?

La réponse d'un État responsable va dans le sens suivant :

Parce que les régions (éloignées et intermédiaires), incluant les territoires ruraux, procurent une contribution essentielle à l'organisation territoriale, à la production économique et culturelle et à la qualité de vie de la société globale. Les régions, composées de petites villes et villages et de vastes espaces naturels :

- assurent une répartition spatiale plus équilibrée de la population et une occupation du territoire en dehors des agglomérations urbaines évitant ainsi la surcharge des centres;

- élargissent l'éventail des choix de modes de vie, de lieux de travail, de production, de décision et de détente;

- favorisent, par l'activité agricole et d'autres formes d'occupation des sols, l'entretien des paysages et l'accès à l'environnement naturel;

- assurent un certain niveau d'autosuffisance agroalimentaire;

- contribuent à préserver et à transmettre un système de valeurs différent ainsi qu'un patrimoine témoin d'une culture pour laquelle la société demeure attachée; 
- répondent à une volonté profonde d'une proportion importante de la population de vivre dans les collectivités régionales et rurales;

- constituent des territoires d'accueil pour la nouvelle économie et des segments de population susceptibles de s'établir en dehors des régions centrales;

- représentent une réserve d'éléments et de potentialités pour résoudre demain les crises des grandes agglomérations urbaines.

L'avenir des régions passe essentiellement par l'affirmation vigoureuse de leur utilité économique, sociale, culturelle et écologique. Il passe aussi par le respect de leur spécificité et de leur droit à la juste part des budgets et des aides gouvernementales.

L'avenir des régions passe essentiellement par l'affirmation vigoureuse de leur utilité économique, sociale, culturelle et écologique.

Pour relever ce défi, les collectivités territoriales doivent pouvoir compter sur de vigoureux programmes de soutien au développement régional. En l'absence d'une politique nationale de développement des territoires, les compétences accrues des collectivités territoriales par la décentralisation risquent d'avoir des effets bien limités sur les efforts de développement économique et social des régions périphériques et intermédiaires aux prises avec des problèmes de survie.

\subsection{Objectif fondamental d'une politique nationale des territoires : réduire les disparités économiques et sociales entre les régions}

Cette politique nationale de développement des territoires se donnera comme axes prioritaires :
- l'amélioration de la compétitivité des économies régionales dans le but de stimuler la création d'emplois durables;

- la diversification des économies régionales par des efforts soutenus et des moyens appropriés en faveur d'activités de deuxième et de troisième transformation et le développement de la nouvelle économie incluant le télétravail. Ceci, tout en poursuivant et consolidant les politiques de mises en valeur des ressources naturelles (agriculture, forêt, mines, pêches) qui demeurent des composantes structurelles des économies régionales.

- le renforcement de la cohésion sociale et de la situation de l'emploi en particulier par la valorisation des ressources humaines, première richesse des régions, dans le but de contrer l'exode des jeunes et le vieillissement prématuré des populations locales. Un territoire qui n'offre pas d'emplois ne peut retenir sa population.

- la reconnaissance du potentiel des «économies identitaires » fondées non seulement sur les ressources naturelles des régions, mais aussi sur leurs caractéristiques sociales, culturelles et environnementales (ceci pour affranchir les régions de l'approche technocratique de métropolisation du territoire québécois, approche qui a pour conséquence de déclasser et d'exclure des pans entiers du Québec intermédiaire et périphérique).

Une politique nationale de développement des territoires favorisera, à l'échelle de chaque région, la mise en place des conditions préalables aux investissements, c'est-à-dire la réunion des conditions qui confèrent un caractère d'attractivité aux territoires par rapport aux attentes et aux besoins des investisseurs potentiels, des travailleurs et des familles. 
Cela requiert une connaissance actualisée de la logique de localisation des entrepreneurs, et une bonne compréhension des tendances économiques et sociales de fond qui modifient les rapports de l'activité économique et sociale avec l'espace. L'évolution structurelle en cours de l'économie procure de nouvelles opportunités de développement pour les régions, opportunités que les collectivités territoriales aux pouvoirs élargis tenteront de saisir avec l'appui des mesures structurantes, des programmes et outils adaptés contenus dans la politique de développement des territoires.

Si la montée de l'économie du savoir, fondée sur la révolution des technologies de l'information et des communications, n'a pas permis jusqu'à maintenant de freiner le mouvement de concentration de l'économie sur quelques grands pôles, un nouvel allié pointe. Il s'agit de l'engouement pour un environnement de qualité. Cette qualité se conjugue avec la pureté de l'air et de l'eau, la beauté et l'authenticité des paysages, l'accès à des espaces naturels protégés, des produits du terroir et bios, des milieux de vie soustraits aux stress et agressions diverses de la grande ville, des habitats financièrement accessibles, des relations sociales étroites, etc. (ces facteurs d'attraction et de rétention des populations locales sont à la base de l'économie résidentielle complémentaire à l'économie de production). Il n'est pas utopique de penser que la combinaison de ces deux «révolutions » - technologique et environnementale puisse désormais permettre, pour nombre

\section{Il y a là une chance à saisir pour plusieurs territoires victimes de la polarisation de l'économie industrielle et de services, d'accueillir un nouveau dynamisme économique et social inscrit dans la société « post-industrielle».}

d'activités, une remise en cause du principe de la concentration et une reconquête de certains territoires qui ont été désertés au profit de quelques grands centres. Il y a là une chance à saisir pour plusieurs territoires victimes de la polarisation de l'économie industrielle et de services, d'accueillir un nouveau dynamisme économique et social inscrit dans la société «post-industrielle». Les régions de la Gaspésie et du Bas-Saint-Laurent présentent à cet égard de nombreux atouts.

L'État ne crée pas le développement, il n'est pas entrepreneur, mais il doit contribuer à créer les conditions propices, c'est-à-dire matérielles, technologiques, financières, humaines et politiques, aux investissements privés et ainsi lutter efficacement contre la fracture entre régions centrales et régions périphériques et intermédiaires.

\subsection{Mettre les territoires en état de se développer}

Plusieurs conditions doivent être réunies pour conférer à un territoire un caractère de compétitivité et d'attractivité à l'égard des investissements privés de production et des familles. En voici quelques-unes :

- un environnement favorable à un esprit d'entreprise;

- une population active adaptée aux besoins de la concurrence;

- des services diversifiés et de qualité offerts aux entreprises et aux personnes (qualité des écoles et des services culturels et de loisirs, services sociaux et de santé, l'ensemble des services de proximité, soutien technique et financier aux entreprises...);

- des aides en capital de démarrage et d'expansion;

- des infrastructures de transport et de communications adaptées aux besoins, incluant le transport fluvial et les télécommunications. On assurera notamment l'accès 
à Internet haute vitesse à l'ensemble des villes et village;

- les aspects qualitatifs et organisationnels (procédures) du support offert aux entreprises ainsi que l'environnement de la production en général (systèmes productifs locaux);

- des programmes de qualification de la maind'œuvre;

- une qualité de vie assurée par la richesse culturelle et patrimoniale, la mise en valeur de l'environnement bâti et la protection des milieux naturels;

- l'attention et la place accordées aux jeunes dans les organismes consultatifs et de prise de décision (conseils municipaux, organismes de développement, clubs d'entrepreneurs...).

Pour faciliter la mise en place d'un environnement capable de garder et d'attirer des investisseurs privés créateurs d'emplois et de nouvelles familles, des investissements publics structurants dans plusieurs domaines et de niveau substantiel devront être réalisés :

- l'amélioration des réseaux de transport terrestre maritime et aérien;

- la formation de la main-d'œuvre dans des secteurs en demande et en émergence;

- les aides financières et techniques à l'installation de nouvelles entreprises;

- l'appui au développement de filières de productions privilégiées;

- l'essor des entreprises de deuxième et de troisième transformation;

- les infrastructures, équipements et services propices à l'implantation des activités de la nouvelle économie;

- la diversification des productions en agriculture, en foresterie et dans les pêches et la promotion de façons de faire écologiques dans ces domaines;
- le développement de nouveaux produits touristiques;

- la disponibilité en logements pour les nouvelles familles ce qui impliquerait plus de souplesse dans l'application du zonage agricole en territoires défavorisés;

- la rénovation des équipements publics des petites villes et des villages;

- le maintien des services de proximité incluant les services médicaux de première ligne, les soins à domicile et la dernière école de village;

- l'extension du réseau de communications électroniques dans toutes les petites villes et communautés rurales, selon diverses technologies, donnant ainsi accès sur tous les points du territoire au service Internet à haut débit comme facteur moderne de soutien aux entreprises et aux travailleurs autonomes (télé-travailleurs) et outil d'information, d'éducation et de divertissement au sein des familles;

- la consolidation des formations de niveaux cégep et universitaire en région, incluant des partenariats avec les milieux économiques et sociaux;

- la mise en place des équipements et services récréatifs et culturels diversifiés et faciles d'accès;

- la protection et la mise en valeur du patrimoine naturel et culturel.

On pourra faire valoir que plusieurs programmes existants poursuivent les objectifs ici énoncés. Oui, mais avec quelle détermination, guidée par quelle stratégie et avec quel niveau de ressources? Les réponses à ces questions dépendent d'abord et avant tout du rôle que l'on croit possible et souhaitable des régions dans le développement global du Québec. 


\section{Exposé des motifs ou les grands enjeux d'une politique de décentralisation}

Dans son acceptation générale, la décentralisation évoque un processus précis. L'État se dessaisit de certaines compétences au profit des collectivités territoriales imputables et autonomes, dont l'administration était exercée jusque-là par ses organes centraux et ses représentants territoriaux.

\subsection{Considérations générales}

La décentralisation implique qu'il y ait :

- des élus locaux disposant d'une libre administration de leurs compétences

- une attribution élargie de responsabilités et de compétences aux collectivités territoriales

- une autonomie financière

- une personnalité morale

La décentralisation est acquise dans la plupart des pays européens et aussi ou à l'ordre du jour dans beaucoup de pays d'Afrique, de l'Amérique du Sud, des Caraïbes et du Pacifique. Et cela, malgré que les processus de décentralisation soient très différents d'un pays à l'autre, non seulement parce qu'ils s'inscrivent dans des cheminements historiques, des cultures et des expériences administratives différentes, mais également parce qu'ils édictent des dispositions formelles variées. Autrement dit, il n'existe pas de modèle de décentralisation qui pourrait être appliqué partout, par simple transposition.

Les pays qui s'engagent dans la décentralisation ont une histoire, une culture, des institutions et des pratiques qui leur sont propres. Les réformes qu'ils conduisent ne pourront être appliquées jusqu'au bout que si elles sont susceptibles d'une appropriation par leur population, c'est-à-dire si elles s'intègrent harmonieusement, par leurs modalités concrètes, dans le contexte politique et culturel, local et national. Pour la plupart des États, l'instauration d'un système de représentation proche des populations est censée favoriser le développement territorial et renforcer la démocratie.

Parce qu'il peut exister une certaine confusion entre les concepts de décentralisation et de déconcentration, il est opportun de bien distinguer le sens de ces deux termes. La décentralisation est un mode d'organisation institutionnelle qui consiste à faire gérer, par des entités administratives élues, les affaires propres d'une collectivité territoriale. Par le principe de la personnalité morale, c'est-à-dire la reconnaissance d'une personnalité juridique propre, des pouvoirs de décisions, justifiés par l'existence de ces affaires propres, sont reconnus à ces entités administratives autres que l'État et non situés par rapport à lui dans une relation hiérarchique. Le processus de décentralisation concerne ainsi les aspects administratifs, financiers et politiques de l'exercice de responsabilités attribuées aux collectivités territoriales.

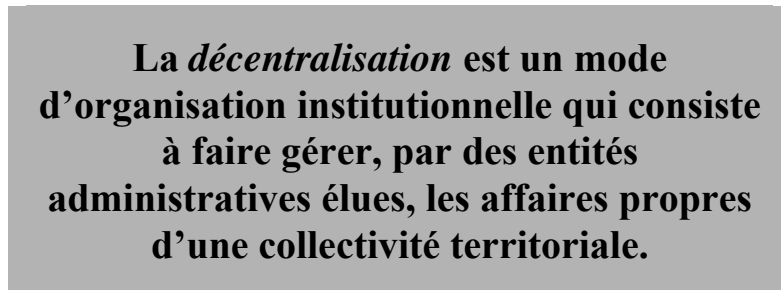

La déconcentration est une technique administrative de délocalisation géographique de la gestion consistant à transférer aux représentants locaux de l'État, répartis sur le territoire national, mais demeurant soumis à l'autorité hiérarchique centrale, le pouvoir de prendre certaines décisions. La régionalisation est une forme de déconcentration. Si décentralisation et déconcentration (régionalisation) vont souvent de pair, elles n'ont pas le même sens. 
La déconcentration est une technique administrative de délocalisation géographique de la gestion consistant à transférer aux représentants locaux de l'État, répartis sur le territoire national, mais demeurant soumis à l'autorité hiérarchique centrale, le pouvoir de prendre certaines décisions.

La décentralisation est une composante fondamentale d'une réforme en profondeur de l'organisation de l'État. Elle est d'une importance telle que dans la plupart des pays où l'on s'est engagé dans cette voie, la décentralisation a donné lieu à des changements constitutionnels. C'est le cas notamment de la France, de l'Italie, de l'Allemagne, de l'Espagne, du Portugal, et de la Roumanie.

\subsection{Principes d'une véritable politique de décentralisation}

Sept principes fondamentaux gouvernent la conception et la mise en œuvre d'une véritable politique de décentralisation :

- Principe de subsidiarité : la répartition des responsabilités et des compétences entre l'État et les collectivités territoriales se fait selon le principe que l'exercice des responsabilités publiques doit, de façon générale, incomber aux autorités les plus proches des citoyens. Seules les responsabilités qui ne peuvent être assumées efficacement au niveau inférieur doivent être réservées à la compétence des autorités au niveau supérieur. Les responsabilités sont ainsi attribuées selon «la bonne échelle territoriale ». L'application de ce principe s'appuiera sur les structures municipales existantes (municipales locales et MRC).

- Principe d'imputabilité des paliers décisionnels décentralisés : les collectivités territoriales s'administrent librement par des conseils élus au suffrage universel direct. Des dispositions de la loi-cadre sur la décentralisation verront à accroître la transparence et l'imputabilité des territoires décentralisés dans la gestion des fonds publics.

- Principe d'autonomie administrative des collectivités territoriales: le principe de la libre administration des collectivités locales sert de fondement à la décentralisation. Les limites de ce principe, définies dans des dispositions de la loi-cadre, s'expliquent par le caractère indivisible du Québec et de la souveraineté nationale. Par ailleurs, l'autonomie administrative des collectivités territoriales doit s'exercer dans le respect des intérêts nationaux, des règles d'une saine gestion administrative et des lois en vigueur.

- Principe d'autonomie financière : il s'agit du principe assurant les collectivités territoriales des moyens et des ressources nécessaires à l'exercice normal de leurs compétences dans le cadre des transferts de compétences de l'État aux collectivités territoriales. L'autonomie financière comprend son corollaire soit le "principe de compensation» signifiant qu'à chaque compétence transférée par l'État, c'est-à-dire qu'à chaque charge financière nouvelle, correspond le transfert d'une ressource suffisante et appropriée destinée à l'assumer.

- Principe d'efficience administrative, d'économie ou de coût nul pour le citoyen : en rapprochant le pouvoir de décision des citoyens, la décentralisation contribuera à alléger le processus décisionnel tout en réduisant les coûts.

- Principe de souplesse dans l'attribution des nouvelles responsabilités : la décentralisation s'appliquera selon une démarche sur mesure d'attribution de nouvelles responsabilités et adaptée aux particularités locales et régionales. Un certain nombre de responsabilités de base nouvelles, communes à chaque municipalité ou MRC, apparaît souhaitable 
pour faire entrer l'ensemble des municipalités et MRC dans cette réforme majeure de l'organisation de l'État et éviter des disparités trop marquées dans le degré d'autonomie des entités municipales.

- Principe de la progressivité dans la mise en œuvre de la réforme institutionnelle: la décentralisation sera mise en œuvre selon une démarche évolutive et progressive respectant en cela le rythme des partenaires et des milieux, des ministères et organismes gouvernementaux.
- Principe de l'effet structurant sur le développement local et régional : un des premiers bénéfices recherchés par la décentralisation est le développement économique, social et culturel des territoires. La décentralisation et les moyens mis à la disposition des collectivités territoriales ainsi que les nouveaux rapports entre l'État et les collectivités doivent être conçus comme un cadre structurel et des outils de nature à stimuler la dynamique de développement des milieux locaux et régionaux.

\section{L'architecture d'une politique de décentralisation pour le Québec}

\section{Selon les pays, la décentralisation repose sur deux, trois, voire quatre niveaux de collectivités territoriales.}

Selon les pays, la décentralisation repose sur deux, trois, voire quatre niveaux de collectivités territoriales. Les collectivités territoriales sont des institutions administratives publiques, distinctes de l'administration de l'État, qui doivent prendre en charge les intérêts de la population d'un territoire précis. Selon le principe de subsidiarité, chaque niveau de collectivité territoriale administre les compétences (responsabilités) qui lui sont le plus appropriées en complémentarité avec celles assumées par les autres paliers de décentralisation et l'état central. Ainsi une municipalité est-elle chargée des intérêts des personnes vivant sur son territoire en regard de plusieurs fonctions : aménagement et urbanisme, voirie, loisirs, protection civile...

$\mathrm{Au}$ Québec, l'historique de l'organisation structurelle de la gestion de l'espace et les réformes réalisées depuis la fin des années 70 (avènement des MRC, redécoupage des régions, fusions municipales et création des Conférences régionales des élus) conduisent à envisager trois ordres de collectivités territoriales à la base de la politique de décentralisation et de régionalisation. Il s'agit de la municipalité de comté (MRC) et de la région (CRÉ).

On reconnaît généralement que le niveau des MRC devrait constituer le lieu privilégié des pouvoirs décentralisés en matière de développement économique et social, consolidant ainsi le rôle de cette structure déjà en place (depuis 1980) et le potentiel de bassin de vie et d'emploi que représente cette assise géographique (quitte à revoir le nombre de MRC et conséquemment la délimitation géographique de certaines d'entre elles). Précisons que selon les termes de leur statut actuel, les régions administratives, gérées par des Conférences régionales des élus dont les membres ne sont pas élus au suffrage universel direct à l'échelle de la région, ne jouissent pas de l'autonomie administrative et de l'imputabilité conférées par l'élection directe. Sans cette imputabilité, la région ne peut être reconnue comme collectivité territoire à part entière dans l'architecture de la décentralisation.

N'étant pas une instance élue (bien que composée principalement d'élus locaux) et ne disposant d'aucun droit d'imposition ou de 
taxation (son budget provient en presque totalité du gouvernement central), la CRÉ, dans sa définition actuelle, ne peut être retenue comme une collectivité territoriale de plein droit, autonome et imputable, au sens de la décentralisation des pouvoirs. Elle apparaît plutôt comme une créature hybride entre la régionalisation et la décentralisation. Ce qui évidemment n'empêche pas qu'elle puisse, en tant que telle, jouer un rôle utile et légitime.

Il faut ici comprendre que du point de vue du projet de décentralisation le remplacement des Conseils régionaux de concertation et de développement par les CRÉ représente un pas dans la bonne direction. Que d'autre part, les protocoles d'entente signés en octobre 2004 entre le gouvernement du Québec et la FQM et l'UMQ d'une part et le gouvernement du Québec et les présidents des CRÉ d'autre part, portent globalement sur «la démarche de décentralisation et de régionalisation des responsabilités » (Protocole d'entente convenu entre le gouvernement du Québec et les présidents des Conférences régionales des élus). Dans sa volonté et ses engagements, de rapprocher le pouvoir de décision des populations et de promouvoir la responsabilisation des instances locales et régionales à l'égard du développement des territoires, le gouvernement adopte simultanément les démarches de décentralisation et de régionalisation, la distinction entre les deux n'étant pas évidente dans les textes des protocoles. Ce qui peut soulever certaines ambiguités et incompréhensions.

Nous sommes d'avis que décentralisation et régionalisation (déconcentration géographique du pouvoir de l'État) peuvent cohabiter et contribuer à l'amélioration des services aux citoyens en termes de diversité, de qualité et de coût. Nous croyons aussi que pour certains services ou fonctions, la régionalisation du pouvoir de l'État aura avantage à être maintenue parallèlement et en complément à la marche de la décentralisation. La reconnais- sance des mérites de la régionalisation ne doit toutefois pas faire oublier que les véritables gains d'une réforme en profondeur du partage des pouvoirs passent par le transfert de responsabilités à des collectivités territoriales imputables pour une plus grande démocratie participative.

Dans plusieurs pays qui se sont dotés d'une organisation décentralisée de l'administration des affaires publiques, la région, comme collectivité territoriale imputable, constitue un niveau majeur, sinon prépondérant dans le partage des responsabilités, des compétences et des ressources financières et humaines. Les récentes réformes constitutionnelles réalisées dans certains pays européens ont pour la plupart confirmé les régions comme collectivités territoriales à part entière et accru leurs responsabilités et compétences là où elles existaient déjà. C'est notamment le cas de la France, de l'Espagne et de l'Italie. L'Espagne et l'Italie ont accordé à leurs régions des compétences, un pouvoir législatif et une autonomie financière qui les rapprochent d'États fédérés comme l'Allemagne et la Belgique. Ils reçoivent fréquemment le qualificatif « d'États régionalisés ».

\section{Dans plusieurs pays qui se sont dotés d'une organisation décentralisée de l'administration des affaires publiques, la région, comme collectivité territoriale imputable, constitue un niveau majeur, sinon prépondérant dans le partage des responsabilités, des compétences et des ressources financières et humaines.}

Dans la perspective d'une reconnaissance des régions du Québec comme véritables collectivités territoriales, celles-ci seraient administrées par un conseil élu au suffrage universel direct; elles recevraient les compétences appropriées à l'échelle de leur territoire d'intervention (en application du principe de subsidiarité), principalement dans les 
domaines des stratégies d'aménagement et de développement, de la planification et de la gestion des ressources naturelles (forêt, pêches, mines, énergies) et des infrastructures, des services et équipements dont la nature et les coûts outrepassent les capacités financières des MRC et des municipalités locales. Des budgets appropriés leur seraient alloués et garantis. Il serait alors question d'une véritable autonomie régionale.

Dans la perspective d'une reconnaissance des régions du Québec comme véritables collectivités territoriales, celles-ci seraient administrées par un conseil élu au suffrage universel direct.
Le statut et le mode de fonctionnement actuels des CRÉ sont plutôt inédits et fragiles. Pourrat-on maintenir longtemps ceux-ci en dehors du processus électoral au suffrage universel direct de leurs membres et les soustraire à un pouvoir de taxation exercé auprès de leurs populations dans le contexte d'une politique de décentralisation dans laquelle la région sera inévitablement appelée à jouer un rôle important, fondé sur une diversité de responsabilités appropriées à l'échelon régional? Imputabilité et autonomie financière réclameront les mêmes prérogatives que celles accordées aux deux autres niveaux de décentralisation, soient les MRC et les municipalités locales.

\section{Adaptation des politiques et programmes gouvernementaux aux spécificités territoriales}

L'autonomie administrative et financière des collectivités territoriales conférée par la décentralisation ne signifie pas l'isolement des administrations décentralisées par rapport à l'État central. Celui-ci demeure un partenaire majeur dans la concrétisation des décisions locales relatives à plusieurs aspects de la vie économique, sociale et culturelle des collectivités. Par le biais de ses politiques, programmes, normes et mesures, le gouvernement central continuera d'exercer un rôle important, parfois déterminant, dans le processus de développement des territoires.

Les principes à la base de la décentralisation obligent désormais l'adaptation des politiques, programmes, normes et mesures du gouvernement central aux particularités locales et régionales. Il ne fait aucun doute que la qualité de l'exercice de l'autonomie territoriale dépendra largement de la qualité de cette adaptation.

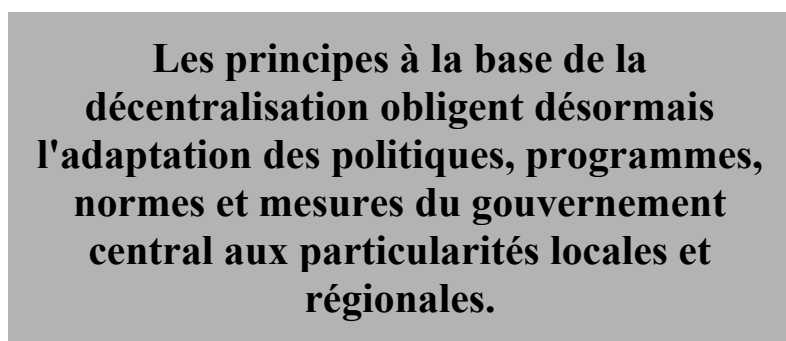

L'adaptation des interventions gouvernementales reposera à la fois sur les modalités d'application de celles-ci et sur l'ouverture et la collaboration dont sauront faire preuve les fonctionnaires de l'État dans leurs relations avec les collectivités territoriales.

La décentralisation ne met pas en péril le caractère unitaire du Québec ni la capacité de décision du gouvernement central à l'égard de ses missions fondamentales et dans ses relations avec les collectivités territoriales. 


\section{Conclusion}

Dans la perspective d'une volonté déterminée et partagée par toutes les parties concernées d'adopter une véritable politique de décentralisation pour le Québec, il est impératif que la réforme qui en découlera ne soit pas uniquement un transfert de responsabilités offert par l'État central, mais le résultat d'une négociation sur des enjeux parfois divergents où les collectivités territoriales auront réellement « conquis » des droits, des pouvoirs et des ressources pour une gouvernance décentralisée au service d'un développement mieux arrimé aux besoins, aux attentes et aux capacités des populations locales et régionales. Au service aussi de la nécessité de permettre à chacune des régions du Québec d'occuper la place qui lui revient dans le contexte d'une économie de plus en plus dématérialisée et mondialisée, marquée par le virage vert et la quête de la qualité de l'habitat et des relations humaines.

\footnotetext{
${ }_{1}^{1}$ Bernard Vachon est professeur à la retraite. Département de géographie. Université du Québec à Montréal.
} 
REVUE TRIMESTRIELLE

\section{RELATIONS INDUSTRIELLES} depuis 1945 par le Département des relations industrielles de I’Université Laval

ARTICLES

Les relations de travail tripartites et l'application des normes minimales du travail au Québec VÉRONIQUE DE TONNANCOUR ET GUYLAINE VALLEE

Jeunes intérimaires et ouvriers permanents en France : quelle solidarité au travail ?

CHRISTIAN PAPINOT

RI/IR EN UGNE

RI/AR est disponible en ligne sur le site Erudit: www.erudit.org/revue/ri

Pour abonnement à la version numérique, contacter Érudit.

Pour consulter les sommaires des numéros publiés, les résumés d'articles ou pour vous abonner à la version papier, visitez notre site Internet: www.riir.ulaval.ca

\section{INDUSTRIAL RELATIONS QUARTERLY REVIEW}

A bilingual quarterly published since 1945 by the Industrial Relations Department, Université Laval

\section{ARTICLES}

Fast Food Work: An Empirical

Perspective of Ideal Employees

ANTHONY M. GOULD

Do Unions Affect Pay Methods of Canadian Firms? A Longitudinal Study

RICHARD J. LONG AND JOHN L. SHIELDS

Income Growth of New Immigrants

in Canada: Evidence from the SLID

RUPA BANERJEE

National Culture and Union

Membership: A Cultural

Cognitive Perspective

RICHARD A. POSTHUMA

\section{RI/IR ON LINE}

$\mathrm{RI} / \mathrm{R}$ is available on line on

Erudit website at:

www.erudit.org/revue/ri

To subscribe to the online version, please contact Erudit.

Visit our website for contents listings, abstracts, or to subscribe to the print edition: www.riir.ulaval.ca

INDUSTRIAL RELATIONS

RELATIONS INDUSTRIELLES

Pavillon J.-A.-DeSève, 1025, avenue des Sciences-Humaines

Bureau 3129, Université Laval, Québec (QC), Canada, G1V OA6

TÉLÉPHONE : (418) 656-2468 TÉLECOPIEUR / FAX : (418) 656-3175

COURRIEL / E-MAIL : relat.ind@rlt.ulaval.ca

wrww.riir.ulaval.ca 